\title{
FATORES RELACIONADOS À OCORRÊNCIA DE EVENTOS ADVERSOS EM PACIENTES IDOSOS INTERNADOS
}

\author{
FACTORS RELATED TO THE OCCURRENCE OF \\ ADVERSE EVENTS IN HOSPITALIZED ELDERLY \\ PATIENTS
}

\section{FACTORES RELACIONADOS A LA OCURRENCIA DE EVENTOS ADVERSOS EN PACIENTES ANCIANOS HOSPITALIZADOS}

\author{
Cristiane Chagas Teixeira ${ }^{1}$ \\ Ana Lúcia Queiroz Bezerra² \\ Thatianny Tanferri de Brito Paranaguá ${ }^{3}$ \\ Valéria Pagotto ${ }^{4}$
}

\begin{abstract}
Como citar este artigo: Teixeira CC, Bezerra ALQ, Paranaguá TTB, Pagotto V. Fatores relacionados à ocorrência de eventos adversos em pacientes idosos internados. Rev baiana enferm. (2018); 32:e25772.

Objetivo: analisar os fatores relacionados à ocorrência de eventos adversos registrados nos prontuários de idosos internados. Método: estudo transversal, conduzido com 260 internações ocorridas na clínica cirúrgica de um hospital de ensino de Goiânia, Goiás, Brasil. Foram realizadas análise descritiva, associação univariada e multivariada. Resultados: verificou-se associação entre presença de acompanhante, tempo de internação igual ou maior que nove dias, uso de seis ou mais medicamentos por dia, uso de hemoderivados e uso de cateter e sonda. O tempo de internação superior a nove dias foi constatado como variável independente de risco para a ocorrência de eventos adversos. Conclusão: a identificação dos fatores relacionados à ocorrência de eventos adversos indica a necessidade de melhorar a qualidade no atendimento, com vistas ao desenvolvimento de competências necessárias para prestar uma assistência livre de danos a idosos internados.
\end{abstract}

Descritores: Enfermagem geriátrica. Qualidade da assistência à saúde. Segurança do paciente.

Objective: analiyze the factors related to the occurrence of adverse events registered in the histories of hospitalized elderly patients. Method: cross-sectional study, involving 260 hospitalizations at the surgical clinic of a teaching hospital in Goiânia, Goiás, Brazil. Descriptive analysis, univariate and multivariate association were performed. Results: associations were found between the presence of a companion, hospitalization for nine days or more, use of six or more medications per day, use of blood derivatives and use of catheter and tube. Hospitalization for more than nine days was an independent variable of risk for the occurrence of adverse events. Conclusion: the identifcation of

\footnotetext{
Enfermeira. Mestre em Enfermagem. Doutoranda pelo Programa de Pós-graduação em Enfermagem da Faculdade de Enfermagem da Universidade Federal de Goiás. Goiânia, Goiás, Brasil. cc-teixeira@hotmail.com

Enfermeira. Doutora em Enfermagem. Professor Associado da Faculdade de Enfermagem da Universidade Federal de Goiás. Goiânia, Goiás, Brasil.

Enfermeira. Doutora em Enfermagem. Professor Adjunto da Faculdade de Ciências da Saúde da Universidade de Brasília. Brasília, Distrito Federal, Brasil.

Enfermeira. Doutora em Enfermagem. Professor Adjunto da Faculdade de Enfermagem da Universidade Federal de Goiás. Goiânia, Goiás, Brasil.
} 
the factors related to the occurrence of adverse events indicates the need to improve the quality of care, with a view to developing the competencies needed to deliver damage-free care to bospitalized elderly.

Descriptors: Geriatric nursing. Quality of health care. Patient safety.

Objetivo: analizar los factores relacionados a la ocurrencia de eventos adversos registrados en los archivos de ancianos internados. Método: estudio trasversal, desarrollado con 260 internaciones ocurridas en la clínica quirúrgica de un hospital de enseñanza de Goiânia, Goiás, Brasil. Fueron aplicados análisis descriptivo, asociación univariada y multivariada. Resultados: fue encontrada asociación entre presencia de acompañante, tiempo de hospitalización igual o superior a nueve días, uso de seis o más medicaciones por día, uso de derivados sanguíneos y uso de catéter y sonda. El tiempo de hospitalización superior a nueve dias fue constatado como variable independiente de riesgo para la ocurrencia de eventos adversos. Conclusión: la identificación de los factores relacionados a la ocurrencia de eventos adversos indica la necesidad de mejorar la calidad en la atención, visando al desarrollo de competencias necesarias para prestar un cuidado libre de daños a ancianos hospitalizados.

Descriptores: Enfermería geriátrica. Calidad de la atención de salud. Seguridad del paciente.

\section{Introdução}

Os eventos adversos advindos do cuidado representam, mundialmente, sérios problemas relacionados à segurança do paciente e constituem importantes indicadores de avaliação da qualidade da assistência. Esses eventos acarretam impactos negativos na saúde dos pacientes, influenciam na perda de confiança na instituição, além de resultarem em repercussões econômicas ${ }^{(1)}$.

De acordo com a Organização Mundial de Saúde (OMS), eventos adversos são incidentes que ocorrem durante a assistência à saúde e resultam em algum tipo de dano ao paciente. Esse dano pode ser físico, social ou psicológico, incluindo doença, lesão não intencional, sofrimento, incapacidade temporária ou permanente e óbito ${ }^{(2)}$.

A proporção de eventos adversos em unidades de internação clínica e cirúrgica é de $56,9 \%{ }^{(3)}$. Especificamente em unidades de internação cirúrgica, estima-se que 18,7\% dos pacientes hospitalizados sejam expostos a um ou mais eventos adversos ${ }^{(4)}$.

Em relação à população acometida pelos eventos adversos, estudos realizados na França e no Canadá demonstraram que os idosos apresentaram uma proporção de 34\% e 64\%, respectivamente, de ocorrência de eventos adversos quando hospitalizados, comparados às populações de outras faixas etárias. Dentre os tipos de eventos, as lesões por pressão, a queda e aqueles relacionados ao processo de medicação foram predominantes ${ }^{(5-6)}$.

Sabe-se que esses eventos aumentam o tempo de permanência dos pacientes em instituições de saúde. Nessa perspectiva, a análise dos eventos adversos evitáveis, ocorridos durante um ano em três hospitais públicos do Rio de Janeiro, constatou 373 dias adicionais de internação, sendo as infecções associadas ao cuidado em saúde responsáveis por 226 dias e as complicações cirúrgicas e/ou anestésicas, por 79 dias $^{(3)}$. Como consequência, a maior parte dos eventos adversos resultaram em danos leves. Entretanto, observou-se o dano grave e o óbito em mais de 2,0\% dos eventos ${ }^{(4)}$.

O maior risco de eventos adversos em idosos pode estar associado às vulnerabilidades, às fragilidades, à alta prevalência de doenças agudas e crônicas e às alterações fisiológicas e homeostáticas próprias da idade. Além disso, esse grupo tende a recorrer com maior frequência aos serviços de saúde. Ademais, as internações e reinternações hospitalares podem tornar-se rotineiras e mais prolongadas ${ }^{(7-8)}$. Soma-se a esses fatores a escassez de profissionais preparados para o atendimento a essa população, podendo aumentar a probabilidade de ocorrência de potenciais danos e agravos à saúde dos idosos hospitalizados $^{(8)}$. 
Frente a esse cenário, e mais especificamente à escassez de publicações envolvendo idosos brasileiros em clínica cirúrgica, é importante conhecer essa realidade para fomentar a reorganização de serviços com vistas à diminuição de danos relacionados à saúde.

Esta pesquisa tem por objetivo analisar os fatores relacionados à ocorrência de eventos adversos registrados nos prontuários de idosos internados.

\section{Método}

Trata-se de uma pesquisa transversal, retrospectiva, realizada na clínica cirúrgica de um hospital de ensino, referência no atendimento de alta complexidade no município de Goiânia, Goiás, Brasil.

A população foi constituída de idosos internados no período de julho a dezembro de 2013. Esse período foi selecionado em decorrência de uma reforma na estrutura física da unidade, no primeiro semestre do ano, que inviabilizou a ocupação total dos leitos para internação de pacientes. Portanto, optou-se por iniciar a investigação no período em que o atendimento foi normalizado. Os dados foram obtidos mediante a análise dos prontuários de 260 idosos internados no referido período.

Como critérios de inclusão foram considerados os indivíduos internados no período proposto com idade $\geq 60$ anos, cujo tempo de internação foi superior a 24 horas. Como critério de exclusão adotou-se o período de internação inferior a 24 horas, por considerar tempo insuficiente para registros relacionados à assistência prestada na unidade.

A coleta de dados foi realizada no mês abril de 2014. Utilizou-se instrumento estruturado, contendo questões referentes às características gerais do paciente e da internação (sexo, idade, tempo de internação, data da admissão e alta hospitalar, comorbidades, presença de acompanhante, tipo de admissão - eletiva/urgência, realização de cirurgia, especialidade médica, diagnóstico da internação, quantidade de drogas utilizadas por dia de internação hospitalar, uso de sondas, drenos, cateteres e outros dispositivos, realização de profilaxia antibiótica e infusão de hemoderivados), e também uma planilha para o levantamento dos possíveis eventos adversos documentados pelos trabalhadores da saúde nos prontuários de idosos (queda, lesão por pressão, exposição a agentes térmicos ou químicos, processos alérgicos a produtos médico-hospitalares, erros de medicação, retirada acidental de sonda, cateter ou dreno, obstrução de sonda, dreno, cateter ou outros dispositivos, fixação inadequada de sonda, dreno ou cateter, deiscência cirúrgica, dor aguda em pré e pós-operatório, infecção hospitalar, falhas técnicas ou de manuseio de equipamentos/materiais, falhas durante técnicas/ procedimentos, hemoderivado, sepse, omissão de cuidado, sinais vitais incompletos, suspensão de cirurgia, evasão, não checagem de cuidados prescritos e falta de materiais médico-hospitalares $)^{(2)}$.

Os dados foram extraídos dos registros da equipe multiprofissional contidos nas diferentes partes do prontuário.

A ocorrência do evento adverso foi a variável de desfecho. A identificação do dano foi direcionada pelo registro explícito da consequência do evento adverso ao idoso durante a internação e sua classificação foi fundamentada na taxonomia para segurança do paciente ${ }^{(2)}$. As variáveis de exposição foram relacionadas ao paciente e à internação.

Para direcionar a análise dos fatores associados, foi registrada a data em que o evento adverso ocorreu, assim como o registro temporal da instalação dos dispositivos tubulares e demais variáveis, assegurando que a variável de exposição estivesse presente antes da ocorrência do evento adverso.

A categorização dos tipos de eventos adversos deu-se conforme a definição da $\mathrm{OMS}^{(2)}$. Após a categorização, os eventos adversos foram analisados e validados por três juízes especialistas na área, de forma independente.

Os dados foram processados pelo software Statistical Package for Social Science, versão 20.0 para Windows, analisados descritivamente, apresentando frequências relativa e absoluta para 
variáveis categóricas e média para as variáveis contínuas. A normalidade das variáveis contínuas foi analisada pelo teste Shapiro-wilk.

Para analisar os fatores relacionados aos eventos adversos foi realizada análise univariada, utilizando o teste Qui-Quadrado para comparar as proporções, e o Odds ratio (OR) como medida de efeito. Para as variáveis contínuas, tais como idade, tempo de internação e número de medicamentos prescritos, considerou-se a média como ponto de corte para a análise dicotômica. Quanto ao tipo de admissão, a comparação decorre da necessidade de avaliar se as internações de caráter de urgência possuem maior risco de eventos adversos em relação às eletivas. As variáveis que obtiveram associações com $\mathrm{p}<0,10$, na análise univariada, foram submetidas à regressão logística múltipla, ajustadas por sexo e idade, considerando-se o nível de significância de 5\%.

Estudo inserido na pesquisa matriz "Análise de Ocorrências de Eventos Adversos em um Hospital da Rede Sentinela na Região Centro-Oeste", aprovada pelo Comitê de Ética do hospital (Protocolo n. 064/2008).

\section{Resultados}

Um total de 260 internações ocorreram na clínica cirúrgica entre julho e dezembro de 2013, correspondendo a 19,9\% de todas as internações no período.

A Tabela 1 apresenta as características dos idosos, os aspectos clínicos da internação e a associação entre as variáveis de exposição e os eventos adversos.

Tabela 1 - Associação univariada entre as variáveis de exposição e a ocorrência de eventos adversos durante as internações de idosos em uma clínica cirúrgica de um hospital de ensino. Goiânia, Goiás, Brasil - $2013(\mathrm{~N}=260)$

\begin{tabular}{|c|c|c|c|c|c|c|}
\hline \multirow[t]{2}{*}{ Variáveis } & \multirow{2}{*}{$\begin{array}{c}\text { Amostra } \\
\text { total } \\
\mathbf{n}\end{array}$} & \multicolumn{2}{|c|}{$\begin{array}{c}\text { Prevalência de } \\
\text { Eventos Adversos }\end{array}$} & \multirow{2}{*}{$\begin{array}{l}\text { Odds } \\
\text { Ratio }\end{array}$} & \multirow{2}{*}{$\begin{array}{c}\text { Intervalo de } \\
\text { Confiança } \\
95 \%\end{array}$} & \multirow[t]{2}{*}{$\mathbf{p}$} \\
\hline & & $\mathbf{n}$ & $\%$ & & & \\
\hline \multicolumn{7}{|l|}{ Faixa Etária } \\
\hline Até 70 anos & 93 & 53 & 56,9 & 0,89 & $0,53-1,48$ & 0,650 \\
\hline 71 anos ou mais & 167 & 100 & 59,8 & & & \\
\hline \multicolumn{7}{|l|}{ Sexo } \\
\hline Masculino & 131 & 83 & 63,3 & 0,68 & $0,42-1,13$ & 0,136 \\
\hline Feminino & 129 & 70 & 54,2 & & & \\
\hline \multicolumn{7}{|l|}{ Comorbidades } \\
\hline $\operatorname{Sim}$ & 178 & 103 & 57,8 & 0,81 & $0,41-1,41$ & 0,451 \\
\hline Não & 73 & 46 & 63,0 & & & \\
\hline \multicolumn{7}{|l|}{ Tipo de Admissão } \\
\hline Urgência & 150 & 95 & 63,3 & 1,55 & $0,94-2,55$ & 0,086 \\
\hline Eletiva & 110 & 58 & 52,7 & & & \\
\hline \multicolumn{7}{|l|}{ Intervenção Cirúrgica } \\
\hline $\operatorname{Sim}$ & 209 & 122 & 58,3 & 0,90 & $0,48-1,69$ & 0,754 \\
\hline Não & 51 & 31 & 60,7 & & & \\
\hline \multirow{2}{*}{\multicolumn{7}{|c|}{$\begin{array}{l}\text { Presença de } \\
\text { Acompanhante }\end{array}$}} \\
\hline & & & & & & \\
\hline $\operatorname{Sim}$ & 142 & 93 & 65,4 & 1,83 & $1,11-3,02$ & 0,042 \\
\hline Não & 49 & 27 & 55,1 & & & \\
\hline \multicolumn{7}{|l|}{ Tempo de Internação } \\
\hline Nove ou mais dias & 67 & 65 & 97,0 & 38,78 & $9,23-162,90$ & 0,000 \\
\hline Um a oito dias & 193 & 88 & 45,5 & & & \\
\hline \multirow{2}{*}{\multicolumn{7}{|c|}{$\begin{array}{l}\text { Número de } \\
\text { Medicamentos }\end{array}$}} \\
\hline & & & & & & \\
\hline 6 ou mais prescrições/dia & 101 & 72 & 71,2 & 2,39 & $1,40-4,07$ & 0,001 \\
\hline 1 a 5 prescrições/dia & 159 & 81 & 50,9 & & & \\
\hline
\end{tabular}


Tabela 1 - Associação univariada entre as variáveis de exposição e a ocorrência de eventos adversos durante as internações de idosos em uma clínica cirúrgica de um hospital de ensino. Goiânia, Goiás, Brasil - $2013(\mathrm{~N}=260)$

(conclusão)

\begin{tabular}{|c|c|c|c|c|c|c|}
\hline \multirow[t]{2}{*}{ Variáveis } & \multirow{2}{*}{$\begin{array}{c}\text { Amostra } \\
\text { total } \\
\mathbf{n}\end{array}$} & \multicolumn{2}{|c|}{$\begin{array}{c}\text { Prevalência de } \\
\text { Eventos Adversos }\end{array}$} & \multirow{2}{*}{$\begin{array}{l}\text { Odds } \\
\text { Ratio }\end{array}$} & \multirow{2}{*}{$\begin{array}{c}\text { Intervalo de } \\
\text { Confiança } \\
95 \%\end{array}$} & \multirow[t]{2}{*}{$\mathbf{p}$} \\
\hline & & $\mathbf{n}$ & $\%$ & & & \\
\hline \multirow{2}{*}{\multicolumn{7}{|c|}{$\begin{array}{l}\text { Infusão de } \\
\text { Hemoderivado }\end{array}$}} \\
\hline & & & & & & \\
\hline Sim & 44 & 35 & 79,5 & 3,23 & $1,48-7,04$ & 0,002 \\
\hline Não & 216 & 118 & 54,6 & & & \\
\hline \multicolumn{7}{|c|}{ Utilização de Cateter } \\
\hline Sim & 227 & 140 & 61,6 & 2,47 & $1,17-5,23$ & 0,015 \\
\hline Não & 33 & 13 & 39,3 & & & \\
\hline \multicolumn{7}{|c|}{ Utilização de Sonda } \\
\hline Sim & 92 & 64 & 69,5 & 2,03 & $1,18-3,47$ & 0,009 \\
\hline Não & 168 & 89 & 52,9 & & & \\
\hline \multicolumn{7}{|c|}{ Utilização de Dreno } \\
\hline Sim & 55 & 35 & 63,6 & 1,29 & $0,70-2,39$ & 0,416 \\
\hline Não & 205 & 118 & 57,5 & & & \\
\hline
\end{tabular}

Fonte: Elaboração própria.

$\mathrm{Na}$ análise univariada, os fatores relacionados à ocorrência dos eventos adversos foram: presença de acompanhante (OR 1,83, IC95\% 1,11-3,02); tempo de internação igual ou superior a nove dias (OR 38,78, IC95\% 9,23-162,90); prescrição de seis ou mais medicamentos por dia (OR 2,39, IC95\% 1,40-4,07); infusão de hemoderivado (OR 3,23, IC95\% 1,48-7,04); uso de cateter (OR 2,47, IC95\% 1,17-5,23) e uso de sonda (OR 2,03, IC95\% 1,18-3,47).

Tabela 2 - Análise de regressão logística múltipla dos fatores relacionados à ocorrência de eventos adversos durante as internações de idosos em uma clínica cirúrgica de um hospital de ensino. Goiânia, Goiás, Brasil - 2013 (N=260)

\begin{tabular}{l|ccccc}
\hline \multirow{2}{*}{ Variáveis } & \multicolumn{4}{|c}{ Odds Ratio (Intervalo de Confiança 95\%) } \\
\cline { 2 - 5 } & Não ajustado & p & Ajustado & p \\
\hline Idade > 70 anos & $0,89(0,53-1,48)$ & 0,650 & $0,66(0,35-1,23)$ & 0,189 \\
Sexo masculino & $0,68(0,42-1,13)$ & 0,136 & $0,65(0,37-1,17)$ & 0,153 \\
Presença de acompanhantes & $1,83(1,11-3,02)$ & 0,042 & $0,85(0,47-1,56)$ & 0,603 \\
Internação > 9 dias & $38,78(9,23-162,9)$ & 0,000 & $34,16(7,72-151,12)$ & $\mathbf{0 , 0 0 0}$ \\
Infusão de hemoderivado & $3,23(1,48-7,04)$ & 0,002 & $1,03(0,38-2,75)$ & 0,959 \\
Utilizar sonda & $2,03(1,18-3,47)$ & 0,009 & $1,05(0,54-2,05)$ & 0,891 \\
Utilizar cateter & $2,47(1,17-5,23)$ & 0,015 & $1,24(0,55-2,78)$ & 0,600 \\
$\geq 5$ medicamentos prescritos & $2,39(1,40-4,07)$ & 0,001 & $1,26(0,67-2,37)$ & 0,472 \\
\hline
\end{tabular}

Fonte: Elaboração própria.

$\mathrm{Na}$ Tabela 2, a análise de regressão logística múltipla indicou uma variável tempo de internação com associação independente, com probabilidade 34 vezes maior de ocorrer eventos adversos entre os idosos com tempo de internação igual ou superior a nove dias.

\section{Discussão}

Pacientes idosos apresentam elevado risco de sofrer eventos adversos durante o período de internação ${ }^{(5-6)}$. A exposição dos idosos aos eventos adversos pode ser explicada pela sua 
condição de saúde no momento da internação, por respostas não promissoras ao tratamento, pelo prolongamento do tempo de internação, pela qualificação dos profissionais para realizar procedimentos em indivíduos com a saúde fragilizada, assim como pelas características inerentes ao processo de envelhecimento, que resultam em dificuldades na tomada de decisão efetiva durante a prática assistencial ${ }^{(7,9)}$.

No contexto da prática assistencial em saúde, estudo apontou que um único paciente pode sofrer múltiplos eventos adversos durante a prestação do cuidado ${ }^{(4)}$. Por isso, a assistência deve ser planejada de acordo com a individualidade de cada paciente e com o conhecimento das características clínicas e epidemiológicas que predispõem à ocorrência dos eventos adversos, na medida em que permitam minimizá-los e/ou eliminá-los ${ }^{(6)}$.

O tempo de internação é comumente visto como fator de risco e influencia na ocorrência de eventos adversos ${ }^{(9)}$. Em instituições de saúde, as consequências desses eventos durante a permanência do paciente idoso são diversas. Cita-se como exemplo o prolongamento do tempo de permanência, incapacidades temporárias ou permanentes, aumento dos custos para as instituições e a sociedade. O resultado mais lesivo é acarretar a morte dos pacientes ${ }^{(1,6)}$.

Neste estudo, o tempo de internação igual ou superior a 9 dias apresentou associação independente, com probabilidade 34 vezes maior de ocorrer eventos adversos quando comparado às internações com período menor que 9 dias.

Os fatores que podem contribuir para o adiamento da alta hospitalar são influenciados pela idade, sexo, condições clínicas do paciente, diagnóstico e assistência prestada, atrasos ou cancelamentos na realização de procedimentos cirúrgicos, desestabilização clínica, tempo de espera de estabilização do quadro clínico, necessidade de atendimento em outros níveis de complexidade, pacientes em antibioticoterapia, dificuldades financeiras, familiares e sociais ${ }^{(9,10)}$.

A literatura aponta que pacientes com um período de internação superior a 15 dias apresentam maior risco nutricional ${ }^{(11)}$, situação que pode ser agravada pela condição de ser idoso. Além disso, a indisponibilidade de recursos por parte da organização e a deficiência no dimensionamento de pessoal também são fatores que podem interferir diretamente no tempo de permanência, por estarem associados à qualidade no atendimento ao paciente. Assim, tempo de permanência significativamente mais longo pode ser indicativo de ineficiência administrativa e/ou baixa qualidade do cuidado em saúde ${ }^{(9,10)}$.

Embora não tenham mantido associação independente após análise de regressão múltipla, a presença de acompanhante, infusão de hemoderivado, uso de dispositivos tubulares e prescrição de seis ou mais medicamentos por dia apresentaram associação estatisticamente significativa na análise univariada.

A presença de acompanhante pode favorecer a participação ativa no cuidado ao idoso, podendo minimizar e/ou eliminar danos desnecessários durante o período de hospitalização ${ }^{(12)}$. No entanto, a presença de acompanhante foi fator associado à presença de eventos adversos. Como essa associação não foi sustentada na análise múltipla, pode-se levantar como hipótese para a ocorrência dessa associação na análise univariada a possibilidade de o enfermeiro delegar alguns cuidados sem a devida orientação. Ressalta-se que o Código de Ética de Enfermagem, em seu artigo 92, pró́be delegar atribuições dos profissionais de enfermagem, previstas na legislação, para acompanhantes e/ou responsáveis pelo paciente ${ }^{(13)}$.

A terapia transfusional é uma intervenção amplamente utilizada no âmbito da saúde que, apesar de salvar vidas ou melhorar uma grave condição, possui riscos inerentes à sua prática, com potencial de ocasionar desfechos clínicos fatais $^{(14)}$. Em idosos, essa opção terapêutica é essencial para o tratamento de inúmeras enfermidades, como em casos de anemias agudas e crônicas, neoplasias, aplasia medular e assistência de emergência ${ }^{(15)}$. Nos Estados Unidos, o registro voluntário de eventos adversos relacionados à hemovigilância, entre os anos de 2010 e 2012, constatou a ocorrência de reações alérgicas em $46,8 \%$ dos casos. Foram consideradas graves ou 
com risco de vida $7,2 \%$ de todas as reações e $0,1 \%$ foram fatais ${ }^{(16)}$. Apesar de reconhecidas as normas para a terapia tranfusional, as principais causas dos eventos com dano ainda estão relacionadas a fatores humanos ${ }^{(14)}$.

Tratando-se do uso dos dispositivos tubulares, independentemente da finalidade, uso inadequado da técnica, do tempo de utilização e habilidade profissional, os riscos para os pacientes podem ser graves. Desta forma, para tornar seguro o uso desses dispositivos e evitar a ocorrência de danos desnecessários aos pacientes durante a assistência prestada, há necessidade de adotar-se processos de trabalho bem estabelecidos e adaptados às características de cada paciente ${ }^{(17)}$.

Considerando que as hospitalizações podem produzir consequências negativas para a saúde dos idosos, o paciente cirúrgico está exposto ao uso de equipamentos invasivos, como cateteres e sondas, o que prolonga o tempo de permanência no leito e a probabilidade de eventos $\operatorname{adversos}^{(18)}$.

A investigação de comorbidades como fator de exposição aos eventos adversos deve ser considerada, pois, além de tornar o idoso fragilizado, essa condição está frequentemente associada a diversas indicações medicamentosas. Estima-se prevalência de 58,4\% a 75,0\% de uso de medicamentos inapropriados aos idosos durante a hospitalização, dependendo do critério utilizado para avaliação ${ }^{(19)}$.

Nessa perspectiva, é importante avaliar a terapia medicamentosa durante a hospitalização, especialmente na ocorrência de prescrições de múltiplas terapias. A polifarmácia contribui para o aumento de reações adversas a medicamentos, interações medicamentosas, efeitos colaterais indesejáveis e redução da adesão ao tratamento ${ }^{(19)}$. Esses eventos podem ser atribuídos à vulnerabilidade dos idosos aos eventos adversos relacionados aos medicamentos ${ }^{(5)}$, o que se deve à maior ocorrência de problemas de saúde, geralmente, de natureza crônica e de maior gravidade.

A ausência e/ou insuficiência de informações sobre os fatores associados aos eventos adversos é determinante e contribui significativamente para a ocorrência de novos eventos ${ }^{(20)}$. Nesse sentido, enfatiza-se a necessidade de avaliar o idoso desde a admissão até a alta, assim como a implementação de estratégias educativas para a notificação, com vistas a promover a capacitação profissional.

No Brasil, o Programa Nacional de Segurança do Paciente (PNSP), instituído em 2013 pelo Ministério da Saúde, pela Portaria n. 529/2013 e pela Resolução da Diretoria Colegiada (RDC) n. 36/2013, representa uma importante iniciativa no âmbito da gestão para a qualificação do cuidado em saúde em todos os estabelecimentos de saúde do território nacional ${ }^{(21)}$.

No tocante à gestão em saúde, o maior desafio consiste em capacitar e/ou profissionalizar os profissionais que ocupam cargos de gestão para ampliar o uso de tecnologias eletrônicas de comunicação e informação, além de reduzir a instabilidade e rotatividade dos gestores na área pública, por implicações político partidárias ${ }^{(22)}$. A superação de dificuldades relacionadas à gestão está diretamente relacionada à eficiência e eficácia dos serviços ofertados pelo sistema de saúde.

Nessa perspectiva, conhecer os fatores relacionados aos eventos adversos em uma população que carece de cuidados seguros e de qualidade, pode direcionar a elaboração de ações educativas para notificação e prevenção de eventos adversos, políticas de gestão e avaliação mais eficazes, tendo como foco o cuidado especializado que garanta o cuidado seguro do paciente idoso hospitalizado.

Como limitações para este estudo, ressalta-se a possibilidade de subnotificações de eventos adversos no prontuário dos idosos, o que pode indicar exposição superior à retratada. Essa análise evidencia a importância do apoio organizacional no desenvolvimento de competências atitudinais nos profissionais de saúde, para compreensão de que os eventos adversos sejam notificados, reportados, mitigados e, então, exploradas todas as formas de prevenção.

A relevância deste estudo está em diagnosticar situações de risco para a ocorrência de eventos adversos em idosos decorrentes do atendimento 
em saúde, o que contribui para a ampliação do conhecimento na área da saúde e enfermagem. A identificação dos fatores relacionados à ocorrência de eventos adversos em idosos permite que gestores, profissionais da saúde e pesquisadores adotem ações voltadas para uma assistência fundamentada na qualidade, no cuidado seguro e na prática baseada em evidências.

\section{Conclusão}

O estudo alcançou o objetivo proposto e evidenciou que as variáveis presença de acompanhante, prescrição de seis ou mais medicamentos por dia, infusão de hemoderivado, uso de cateter e uso de sonda não mantiveram associação na análise múltipla. O tempo de internação maior ou igual a nove dias foi fator de associação independente para a ocorrência de eventos adversos, aumentando o risco de exposição em 34 vezes quando comparado aos pacientes idosos com internação de período inferior a nove dias. A sua divulgação amplia o conhecimento dos profissionais e da comunidade em geral na compreensão dos fatores que contribuem para eventos adversos que podem gerar alterações psicológicas e comportamentais em idosos hospitalizados.

Conclui-se que a identificação dos fatores relacionados à ocorrência de eventos adversos indica a necessidade de melhorar a qualidade no atendimento, com vistas ao desenvolvimento de competências necessárias para prestar uma assistência livre de danos a idosos internados.

Espera-se que os resultados levantados subsidiem a tomada de decisão em priorizar o cuidado seguro e a qualidade no atendimento, visando o desenvolvimento de competências fundamentais para prestar uma assistência livre de danos a idosos hospitalizados. Estudos voltados para essa temática fazem-se necessários, a fim de apontar medidas preventivas e corretivas que transformem essa realidade e assegurem a incorporação de evidências científicas na prática dos profissionais de saúde, considerando as especificidades desse grupo etário.

\section{Colaborações:}

1. concepção, projeto, análise e interpretação dos dados: Cristiane Chagas Teixeira, Ana Lúcia Queiroz Bezerra, Thatianny Tanferri de Brito Paranaguá e Valéria Pagotto.

2. redação do artigo e revisão crítica relevante do conteúdo intelectual: Cristiane Chagas Teixeira, Ana Lúcia Queiroz Bezerra, Thatianny Tanferri de Brito Paranaguá e Valéria Pagotto.

3. aprovação final da versão a ser publicada: Cristiane Chagas Teixeira e Ana Lúcia Queiroz Bezerra.

\section{Referências}

1. Hoogervorst-Schilp J, Langelaan M, Spreeuwenberg $\mathrm{P}$, de Bruijne MC, Wagner C. Excess length of stay and economic consequences of adverse events in Dutch hospital patients. BMC Health Serv Res. 2015;15:531.

2. World Alliance for Patient Safety. Taxonomy. The conceptual framework for the international classification for patient safety: final technical report. Geneva; 2009.

3. Mendes W, Pavão ALB, Martins M, Moura MLO, Travassos C. Características de eventos adversos evitáveis em hospitais do Rio de Janeiro. Rev Assoc Med Bras [Internet]. 2013 [cited 2016 Dec 12];59(5):421-8. Available from: http://www.scielo.br/scielo.php?script=sci_ arttext\&pid=\$0104-42302013000500006

4. Paranaguá TTB, Bezerra ALQ, Silva AEBC, Azevedo Filho FM. Prevalence of no harm incidents and adverse events in a surgical clinic. Acta Paul Enferm. 2013;26(3):256-62.

5. Ackroyd-Stolarz S, Bowles SK, Giffin L. Validating administrative data for the detection of adverse events in older hospitalized patients. Drug Healthc Patient Saf. 2014;6:101-8.

6. Dupouy J, Moulis G, Tubery M, Ecoiffier M, Sommet A, Poutrain JC, et al. Which adverse events are related to health care during hospitalization in elderly inpatients? Int J Med Sci. 2013;10(9):1224-30.

7. Buurman BM, Frenkel WJ, Abu-Hanna A, Parlevliet JL, de Rooij SE. Acute and chronic diseases as part of multimorbidity in acutely hospitalized older patients. Eur J Intern Med. 2016;27:68-75. 
8. Carretta MB, Bettinelli LA, Erdmann AL, Higashi GDC, Santos JLG. Compreendendo o significado do ser idoso vivenciando sua autonomia na hospitalização. Rev Rene. 2013;14(2):331-40.

9. Ceballos-Acevedo T, Velásquez-Restrepo PA, JaénPosada JS. Duración de la estancia hospitalaria. Metodologías para su intervención. Rev Gerenc Polít Salud. 2014;13(27):274-95.

10. Silva AMN, Souza EFD, Barbosa TLA, Silva CSO, Gomes LMX. Fatores que contribuem para o tempo de internação prolongada no ambiente hospitalar. J Res: Fundam care. 2014;6(4):1590-600.

11. Duarte A, Marques AR, Sallet LHB, Colpo E. Nutritional risk in hospitalized patients during hospital stay. NutrClín Diet Hosp. 2016;36(3):146-52.

12. Wolff JL, Boyd CM, Gitlin LN, Bruce ML, Roter DL. Going it together: persistence of older adults' accompaniment to physician visits by a family companion. J Am Geriatr Soc. 2012;60(1):106-12.

13. Conselho Federal de Enfermagem. Resolução n. 564/2017. Aprova o novo Código de Ética dos Profissionais de Enfermagem [Internet]. 2017 [cited 2018 Jul 23]. Available from: http://www.cofen.gov.br/ resolucao-cofen-no-5642017_59145.html

14. Bolton-Maggs PHB, Cohen H. Serious Hazards of Transfusion (SHOT) haemovigilance and progress is improving transfusion safety. $\mathrm{Br} \mathrm{J}$ Haematol. 2013;163(3):303-14.

15. Pornkuna R, Wongkhantee $S$, Jinathongthai $S$, Shimogawa S, Takemoto S. Effect of Blood Transfusion on Supportive Therapy of Elderly Patients at Kumamoto, Japan as Compared with Khon Kaen, Thailand. J Hematol Thrombo Dis. 2014;2(3):152.
16. Harvey AR, Basavaraju SV, Chung KW, Kuehnert MJ. Transfusion-related adverse reactions reported to the National Healthcare Safety Network Hemovigilance Module, United States, 2010 to 2012. Transfusion. 2015;55(4):709-18.

17. Silva AEBC, Laselva CR, Carrara D, Perini E, Pinto GRS, Sousa MRG, et al. Erros de conexão: práticas seguras e riscos na administração de soluções por sondas enterais e cateteres vasculares. Bol ISMP Brasil [Internet]. 2013 [cited 2016 Nov 30];2(3):1-4. Available from: http://www.ismp-brasil.org/site/ wp-content/uploads/2015/07/V2N3.pdf

18. Vendites S, Almada-Filho CM, Minossi JG. Aspectos gerais da avaliação pré-operatória do paciente idoso cirúrgico. Arq Bras Cir Dig. 2010;23(3):173-82.

19. Tosato M, Landi F, Martone AM, Cherubini A, Corsonello A, Volpato S, et al. Potentially inappropriate drug use among hospitalised older adults: results from the CRIME study. Age Ageing. 2014;43(6):767-73.

20. Loke YK. Lack of clarity in reports of adverse events: is there any harm? Pain. 2013;154(2):183-4.

21. Brasil. Ministério da Saúde. Agência Nacional de Vigilância Sanitária. Portaria n. 529, de 1 de abril de 2013. Institui o Programa Nacional de Segurança do Paciente. Brasília; 2013.

22. Lorenzetti J, Lanzoni GMM, Assuiti LFC, Pires DEP, Ramos FRS. Gestão em saúde no Brasil: diálogo com gestores públicos e privados. Texto Contexto Enferm. 2014;23(2):417-25.

Recebido: 28 de fevereiro de 2018

Aprovado: 14 de agosto de 2018

Publicado: 16 de outubro de 2018

A Revista Baiana de Enfermagem utiliza a Licença Creative Commons - Atribuição-NãoComercial 4.0 Internacional. https://creativecommons.org/licenses/by-nc/4.0/ Este artigo é de acesso aberto distribuído sob os termos da Licença Creative Commons (CC BY-NC). Esta licença permite que outros remixem, adaptem e criem a partir do seu trabalho para fins não comerciais. Embora os novos trabalhos tenham de lhe atribuir o devido crédito e não possam ser usados para fins comerciais, os usuários não têm de licenciar esses trabalhos derivados sob os mesmos termos. 